\title{
Article
}

\section{MET Amplification in Non-Small Cell Lung Cancer (NSCLC)-A Consecutive Evaluation Using Next- Generation Sequencing (NGS) in a Real-World Setting}

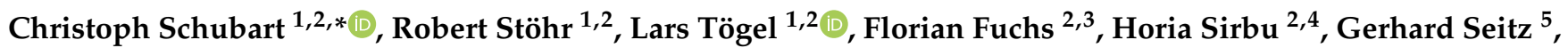 \\ Ruth Seggewiss-Bernhardt ${ }^{6}$, Rumo Leistner ${ }^{7}$, William Sterlacci ${ }^{8}$, Michael Vieth ${ }^{8}$, Christoph Seidl ${ }^{9,10}$, \\ Michael Mugler 9,10, Markus Kapp ${ }^{11}$, Wolfgang Hohenforst-Schmidt ${ }^{12}$, Arndt Hartmann ${ }^{1,2}$, Florian Haller ${ }^{1,2}$ \\ and Ramona Erber ${ }^{1,2}$ (D)
}

Citation: Schubart, C.; Stöhr, R.; Tögel, L.; Fuchs, F.; Sirbu, H.; Seitz, G.; Seggewiss-Bernhardt, R.; Leistner, R.; Sterlacci, W.; Vieth, M.; et al. MET Amplification in Non-Small Cell Lung Cancer (NSCLC)-A

Consecutive Evaluation Using Next-Generation Sequencing (NGS) in a Real-World Setting. Cancers 2021, 13, 5023. https://doi.org/10.3390/ cancers13195023

Academic Editor: Roberta Alfieri

Received: 12 August 2021

Accepted: 27 September 2021

Published: 7 October 2021

Publisher's Note: MDPI stays neutral with regard to jurisdictional claims in published maps and institutional affiliations.

Copyright: (c) 2021 by the authors. Licensee MDPI, Basel, Switzerland. This article is an open access article distributed under the terms and conditions of the Creative Commons Attribution (CC BY) license (https:// creativecommons.org/licenses/by/ $4.0 /)$.
1 Institute of Pathology, University Hospital Erlangen, Friedrich-Alexander-Universität Erlangen-Nürnberg (FAU), 91054 Erlangen, Germany; robert.stoehr@uk-erlangen.de (R.S.); lars.toegel@uk-erlangen.de (L.T.); arndt.hartmann@uk-erlangen.de (A.H.);

florian.haller@uk-erlangen.de (F.H.); ramona.erber@uk-erlangen.de (R.E.)

2 Comprehensive Cancer Center Erlangen-EMN (CCC ER-EMN), 91054 Erlangen, Germany; florian.fuchs@uk-erlangen.de (F.F.); Horia.Sirbu@uk-erlangen.de (H.S.)

3 Department of Medicine 1, University Hospital Erlangen, Friedrich-Alexander-Universität Erlangen-Nürnberg (FAU), 91054 Erlangen, Germany

4 Department of Thoracic Surgery, University Hospital Erlangen, Friedrich-Alexander-Universität Erlangen-Nürnberg (FAU), 91054 Erlangen, Germany

5 Institute of Pathology, Neuropathology, Molecular Diagnostics and Cytology, Klinikum Bamberg, Sozialstiftung Bamberg, 96049 Bamberg, Germany; gerhard.seitz@sozialstiftung-bamberg.de

6 Department of Medicine 5, Klinikum Bamberg, Sozialstiftung Bamberg, 96049 Bamberg, Germany; ruth.seggewiss-bernhardt@sozialstiftung-bamberg.de

7 Department of Medicine 4, Klinikum Bamberg, Sozialstiftung Bamberg, 96049 Bamberg, Germany; rumo.leistner@sozialstiftung-bamberg.de

8 Institute of Pathology, Friedrich-Alexander-Universität Erlangen-Nürnberg (FAU), Klinikum Bayreuth, 95445 Bayreuth, Germany; william.sterlacci@klinikum-bayreuth.de (W.S.); michael.vieth@klinikum-bayreuth.de (M.V.)

9 Diagnosticum, Pathology and Cytology, 95032 Hof, Germany; seidl@diagnosticum.eu (C.S.); mugler@diagnosticum.eu (M.M.)

10 Diagnosticum, Laboratory Medicine, Microbiology, Pathology, Human Genetics, 09221 Neukirchen, Germany

11 Department of Gastroenterology, Hepatology and Infectiology, Section Hematology \& Oncology, Sana Klinikum Hof, 95032 Hof, Germany; Markus.Kapp@Sana.de

12 Department of Cardiology, Sana Klinikum Hof, 95032 Hof, Germany; Wolfgang.Hohenforst-Schmidt@Sana.de

* Correspondence: christoph.schubart@uk-erlangen.de

Simple Summary: Lung cancer has a high incidence and affects both men and women. Targeted therapy options directed at certain mutant proteins, and which avoid systemic chemotherapy are already available and emerging. The gene mesenchymal epithelial transition (MET), encoding a receptor tyrosine kinase protein, is amplified in a subpopulation of lung cancer patients. The aim of our consecutive study was to assess whether next-generation sequencing (NGS) is a reliable method for the detection of MET gene copy number. Our study confirmed that NGS is able to detect cases harboring a high-level MET gene amplification but is unreliable and fails to detect the various levels of MET gene amplification. Therefore, NGS cannot replace the gold standard method of fluorescence in situ hybridization for the detection of MET gene copy number.

Abstract: In non-small cell lung cancer (NSCLC), approximately 1-3\% of cases harbor an increased gene copy number (GCN) of the MET gene. This alteration can be due to de novo amplification of the MET gene or can represent a secondary resistance mechanism in response to targeted therapies. To date, the gold standard method to evaluate the GCN of MET is fluorescence in situ hybridization (FISH). However, next-generation sequencing (NGS) is becoming more relevant to optimize therapy by revealing the mutational profile of each NSCLC. Using evaluable $n=205$ NSCLC cases of a consecutive cohort, this study addressed the question of whether an amplicon based NGS assay 
can completely replace the FISH method regarding the classification of MET GCN status. Out of the 205 evaluable cases, only $n=9$ cases (43.7\%) of $n=16$ high-level MET amplified cases assessed by FISH were classified as amplified by NGS. Cases harboring a MET GCN > 10 showed the best concordance when comparing FISH versus NGS (80\%). This study confirms that an amplicon-based NGS assessment of the MET GCN detects high-level MET amplified cases harboring a MET GCN > 10 but fails to detect the various facets of $M E T$ gene amplification in the context of a therapy-induced resistance mechanism.

Keywords: NSCLC; next-generation sequencing; MET; fluorescence in situ hybridization; amplification; precision medicine; MET inhibitor; resistance mechanism

\section{Introduction}

In solid tumors, driver mutations frequently occur in receptor tyrosine kinases (RTKs) [1]. The proto-oncogene MET (mesenchymal epithelial transition, c-MET or hepatocyte growth factor receptor, HGFR) encodes an RTK protein, which is mainly expressed on the surface of epithelial cells and is physiologically activated by the binding of the HGF ligand (summarized in $[2,3]$ ). Binding of the ligand induces tyrosine phosphorylation, leading to homodimerization of the MET receptor. The signaling cascade leads to a change in gene expression, causing cell cycle progression, cell proliferation and increased motility and invasion [2].

In a variety of malignant tumors, the $M E T$ gene represents one of the drivers of tumorigenesis due to genetic aberrations, including germline or somatic point mutations, splice-site mutations leading to the skipping of exon 14 (MET exon 14 skipping) or gene amplification [2,4].

The genetic architecture of the MET gene locus is organized in a ladder-like structure characterized by many inverted repeats and therefore resembles an area of common chromosomal fragile sites [5]. These sites are susceptible to genetic aberrations, including gene amplifications. Amplification of the MET gene mainly occurs in lung cancer patients but can also develop in adenocarcinomas of the gastroesophageal junction $(3.3 \%)$ or in glioblastoma (1.7\%) [6].

The necessity of MET GCN evaluation in advanced NSCLC is apparent since METamplified (non-squamous) NSCLCs has shown significantly poorer prognosis in previous studies [7,8]. Regarding the amplification of the MET gene, a high-level amplification of $M E T$ can be found only in a low number of TKI-naïve NSCLC patients with frequencies ranging from 2.0-4.0\% [6,9-11]. Notably, the definition of high-level amplified MET varies between studies and the test method employed.

Amplification of the MET gene can be classified as a gain in the gene copy number (GCN), i.e., the $M E T$ gene is multiplied in relation to centromere 7 (CEN7) or in comparison to normal, healthy tissue. In addition, it can be classified as amplified due to polysomy, i.e., the $M E T$ gene and the centromere CEN7 are both increased in number [12,13]. Of clinical importance, these MET gene aberrations can occur either de novo as an early event in tumorigenesis or as an acquired resistance mechanism due to the use of EGFR tyrosine kinase inhibitors $[9,10,14,15]$. More recent data also indicate that the use of nextgeneration ALK inhibitors induces such a resistance phenotype characterized by MET gene amplification [16]. The amplification itself can be targeted with multikinase or MET-specific inhibitors to improve the outcome [7,14,17]. In 2011, the first efficient response against $M E T$-amplified lung cancer using a tyrosine kinase inhibitor (crizotinib) was reported in women harboring a high-level MET amplification, determined by FISH (MET/CEN7 ratio $>5$ ) [17]. There are only limited data concerning intermediate- to low-level amplification of the MET gene and the associated efficiency of a drug response. In 2014, it was shown that a patient suffering from NSCLC harboring low-level amplification of the MET gene (i.e., $M E T / C E N 7 \geq 1.8$ ratio $\leq 2.2, n=1$ ) had no response to the tyrosine kinase inhibitor 
crizotinib. In contrast, patients with tumors harboring an intermediate amplification (i.e., $M E T / C E N 7$ ratio $>2.2$ to $<5, n=6$ ) of the $M E T$ gene showed a durable response [18].

Capmatinib, another MET tyrosine kinase inhibitor, showed high antitumor activity against MET exon 14-mutated tumors of naïve, untreated NSCLC patients. In addition, it was especially effective against high-level MET amplified tumors (MET GCN $\geq 10$ ) compared to low-level amplified tumors [19].

In a recent study, the authors claimed that MET gene amplification is not always a result of treatment but can pre-exist and be selected for during TKI treatment [20]. The use of a combinatory regimen including EGFR tyrosine kinase inhibitors and a MET inhibitor could be a successful strategy in the near future if the resulting toxicity is well tolerated.

Hence, the assessment of MET copy number changes should be an integral part of the diagnostic workup in advanced NSCLC. For the evaluation of the MET amplification status, fluorescence in situ hybridization (FISH) is an adequate and well-established method that captures the various facets of MET gene amplification, including "true" high-level MET gene amplified cases characterized by a high $M E T$ GCN ( $\geq 6$ per cell) without concomitant polysomy (i.e., a high $M E T / C E N 7$ ratio).

According to various studies, multigene testing for nonsynonymous mutations, translocations and copy number variations of a variety of genes is recommended to identify optimal treatment options for patients with advanced tumors, including NSCLC [21-23]. Next-generation sequencing (NGS), which does not reveal genetic aberrations of only one but multiple genes at the same time, represents a tissue-sparing alternative for the detection of various genetic aberrations. Another advantageous aspect of the NGS approach is the detection of MET exon 14 skipping aberrations, which cannot be detected by FISH but is of high clinical relevance [4]. Various methods exist to define the copy number of a gene by NGS summarized in [24].

The present study utilized consecutive, real-world data to investigate whether a multiplex, PCR amplicon-based NGS-based determination of the MET GCN is able to replace the FISH-based approach. In addition, the present study aimed to investigate whether an NGS-based determination of the MET GCN status detects all the various facets of $M E T$ amplification.

\section{Materials and Methods}

\subsection{Cohort}

FFPE specimens of $n=327$ consecutive NSCLC cases diagnosed at the Institute of Pathology of the University Hospital Erlangen, Germany, between July 2016 and May 2018 were included in this study. All cases were therapy-naïve except for one patient who had undergone EGFR TKI therapy (Case \#3) for one year. Of a total of $n=327$ samples, $n=205$ samples could be analyzed by next-generation sequencing (NGS) and fluorescence in situ hybridization (FISH) for genetic aberrations, including the status of the MET gene copy number (GCN) (also see results Section 3.1). Cases that were not analyzed by MET FISH during routine diagnostics were retrospectively analyzed using MET FISH. The various analyses were performed double-blinded. Ethical approval was obtained by the local ethics committee (85_17B).

\subsection{MET GCN Detection by NGS}

H\&E staining was performed to confirm the diagnosis of NSCLC and to estimate the tumor cell content. After microdissection of the tumor tissue, DNA was isolated using standard techniques. Regions of interest, including the MET gene, were enriched and amplified according to the manufacturers' instructions, using a multiplex, PCR ampliconbased 15-gene panel (TruSight Tumor 15, TST15 Illumina, Inc., San Diego, CA, USA). In detail, the regions of interest such as the MET gene or the ERBB2 gene were amplified using tagged, gene specific primers followed by target indexing. After this, libraries were cleaned up and the quality was checked via agarose gels or with the aid of a tape station. Finally, the various libraries were pooled and were run on a MiSeq, respectively (Illumina). The 
obtained sequences (.fastq files) were bioinformatically analyzed using the TruSight Tumor 15 application in the BaseSpace Sequence Hub (Illumina) and aligned to the reference sequence hg19. Molecular aberrations, including point mutations, deletions and insertions, were annotated and described using standard HGVS nomenclature. In addition, changes in gene copy numbers were determined by the CRAFT copy number variant caller (v1.0.0.12) algorithm incorporated in the TST15 application. This algorithm is designed for tumor samples without matched normal controls and can detect amplifications/deletions in three genes (EGFR, ERBB2 and MET) above a 1.6-fold change.

\subsection{MET GCN Detection by FISH}

The MET FISH protocol, established in-house for routine diagnostics, was performed on 1-2 $\mu \mathrm{m}$-thick freshly cut sections from FFPE NSCLC tumor blocks using a MET dualcolor probe (ZytoLight ${ }^{\circledR}$ SPEC MET/CEN7 Dual Color Probe, Z-2087-50, ZytoVision GmbH, Bremerhaven, Germany) according to the manufacturers' standard protocol (ZytoLight FISH-Tissue Implementation Kit, Z-2028-5, ZytoVision $\mathrm{GmbH}$ ) and the routine in-house standards. Pepsin digestion was performed at $37^{\circ} \mathrm{C}$ for $9 \mathrm{~min}$, first hybridization at $75^{\circ} \mathrm{C}$ for $10 \mathrm{~min}$ and the second step at $37^{\circ} \mathrm{C}$ overnight. The MET Dual Color Probe used comprises one orange fluorochrome (ZyOrange)(ZytoVision $\mathrm{GmbH}$, Bremerhaven, Germany) directly labeled CEN7 probe binding specific to the alpha satellite centromeric region of chromosome 7 (D7Z1) and one green labeled (ZyGreen) (ZytoVision GmbH, Bremerhaven, Germany) probe binding the MET gene located at 7q31.2.

The MET FISH status was analyzed, blinded to NGS data, using a Leica fluorescence microscope at $1000 \times$ magnification $(100 \times$ oil objective, Leica Microsystems $\mathrm{GmbH}$, Wetzlar, Germany). A DAPI filter was used to visualize nuclei, and a double-bandpass filter (green/orange) was used to quantify MET and CEN7 signals. After reviewing the H\&E slide of each NSCLC case and reidentifying the tumor cells in DAPI, 50 nonoverlapping tumor nuclei were evaluated, and both green and orange signals per nucleus were counted to determine the mean GCN of $M E T$ and CEN7, respectively. Cases were considered invalid if signals were hardly detectable or missing or if background staining or autofluorescence was too strong. The MET gene copy number status was classified into four groups according to Schildhaus et al. (high-, intermediate- and low-level amplification or normal, nonamplified) [13]. Tumor cells harboring CEN7 signals on average $\geq 3.6$ were classified as polysomic [25].

\subsection{Statistical Analysis}

Venn diagrams were generated using the BioVenn-Web tool [26]. The Oncoprinter illustration option of cBioPortal was used to correlate mutations according to the sex and age of the patients $[27,28]$.

\section{Results}

3.1. Comparison of the MET GCN of the NSCLC Cohort Using Two Different Methods: FISH versus NGS

Out of $n=327$ consecutive NSCLC cases in total, $n=107$ cases could not be analyzed via FISH due to an insufficient quality of the fluorescence signal, limited amount of material or lack of the corresponding material (formalin-fixed and paraffin-embedded (FFPE) material from external pathologies). Out of the overall cohort ( $n=327$ NSCLC cases), $n=23$ cases could not be analyzed via NGS due to a limited amount of material or due to insufficient coverage of the $M E T$ gene locus (minimum amplicon count of $>200$ ). Overall, $n=205$ NSCLC cases could be directly compared using both NGS and FISH regarding the determination of the MET GCN status.

Figure 1 depicts the various facets of MET gene amplification assessed by FISH in the present study. High-level MET amplified cases, characterized by a high MET/CEN7 ratio $(\geq 2.0)$ or cluster formation (Figure 1a,b), and high-level MET amplified cases, characterized 
by a high MET GCN $(\geq 6.0)$, accompanied by a polysomic state of the cells, were detected in the present study cohort (Figure 1c).

a

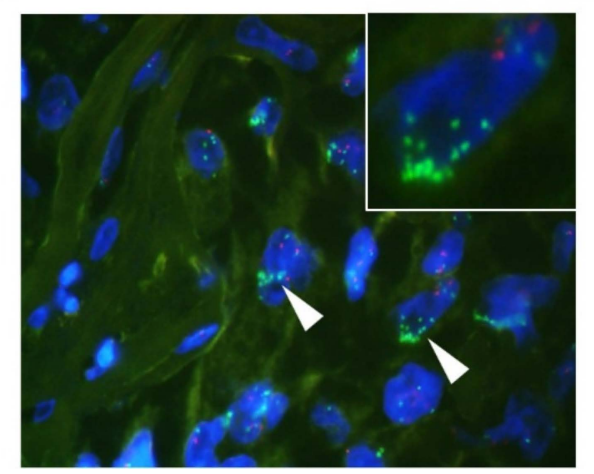

b

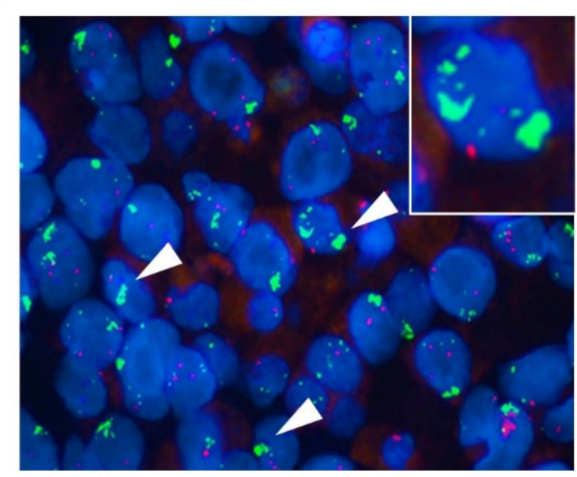

C

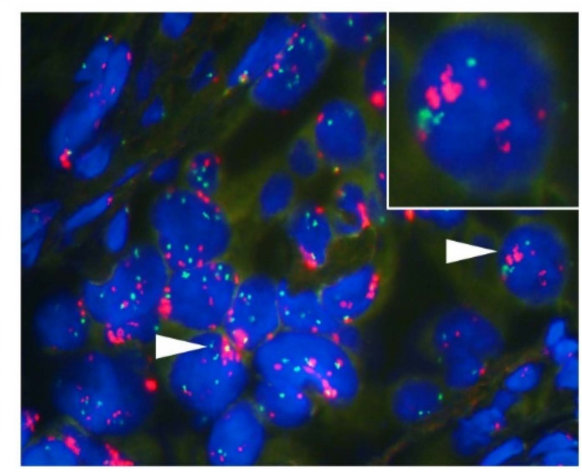

Figure 1. Overview of the various facets of a MET amplification assessed by FISH (each $1000 \times$ magnification). Representative fluorescence images showing a high-level MET gene amplification characterized by (a) a high MET GCN, (b) clusterformation (c) or accompanied polysomy. Green signals represent the MET probe, red signals represent the CEN7 probe. White arrows indicate the relevant areas. Images in the upper right are magnified areas of the analyzed slide.

In the cohort of $n=205$ evaluable cases, $n=42$ cases showed an aberrant GCN of MET assessed by FISH (Table 1). These cases harbored various levels of MET gene amplification ranging from low to high levels. Of the $n=42$ cases, $n=24$ cases were characterized by polysomy (57.0\%). Focusing on the clinically relevant high-level MET amplified cases assessed by FISH, $n=16$ cases of $n=42$ cases showed a $M E T / C E N 7$ ratio $\geq 2.0$ or an average MET GCN per cell $\geq 6.0$ or $\geq 10 \%$ of tumor cells containing $\geq 15$ MET signals (Table 1 and Figure 2a). In the cohort of $n=205$ cases, $n=9$ cases harbored a "true" high-level MET gene amplification assessed by FISH, i.e., cases without a concomitant polysomy (4.4\%).

Table 1. Overview of the MET amplified cases assessed by FISH and NGS. Table showing cases \#1-43 harboring an amplification of the MET gene determined by FISH or NGS. Cases are arranged according to their FISH classification, descending from high-level amplified cases to low-level amplified cases and descending from cases with the highest $M E T$ GCN to the lowest. The columns show the various FISH parameters (MET/CEN7 ratio, MET GCN, CEN7 GCN, MET status and polysomy) as well as the NGS status. Cases which were classified as amplified by the NGS approach are highlighted in green.

\begin{tabular}{ccccccc}
\hline & & & FISH & & NGS \\
\hline Case-ID & MET/CEN7 Ratio & MET GCN & CEN7 GCN & MET Status & Polysomy & NGS Status \\
\hline$\# 1$ & $>5.00$ & $>10.00$ & 2.00 & $\begin{array}{c}\text { High-level } \\
\text { amplification } \\
\text { High-level }\end{array}$ & No & No \\
amplification \\
H3
\end{tabular}


Table 1. Cont.

\begin{tabular}{|c|c|c|c|c|c|c|}
\hline & & & FISH & & & NGS \\
\hline Case-ID & MET/CEN7 Ratio & MET GCN & CEN7 GCN & MET Status & Polysomy & NGS Status \\
\hline$\# 10$ & 1.32 & 6.00 & 5.00 & $\begin{array}{l}\text { High-level } \\
\text { amplification }\end{array}$ & Yes & Normal \\
\hline$\# 11$ & 1.50 & 6.00 & 4.00 & $\begin{array}{l}\text { High-level } \\
\text { amplification }\end{array}$ & Yes & Normal \\
\hline$\# 12$ & 1.30 & 6.00 & 4.50 & $\begin{array}{l}\text { High-level } \\
\text { amplification }\end{array}$ & Yes & Normal \\
\hline$\# 13$ & 2.50 & 5.00 & 2.00 & $\begin{array}{l}\text { High-level } \\
\text { amplification }\end{array}$ & No & Normal \\
\hline$\# 14$ & 3.00 & 4.50 & 1.50 & $\begin{array}{l}\text { High-level } \\
\text { amplification }\end{array}$ & No & Normal \\
\hline$\# 15$ & 2.70 & 4.40 & 1.60 & $\begin{array}{c}\text { High-level } \\
\text { amplification }\end{array}$ & No & Amplified \\
\hline$\# 16$ & 2.00 & 4.00 & 2.00 & $\begin{array}{c}\text { High-level } \\
\text { amplification }\end{array}$ & No & Normal \\
\hline$\# 17$ & 1.38 & 5.50 & 4.00 & $\begin{array}{l}\text { Intermediate-level } \\
\text { amplification }\end{array}$ & Yes & Normal \\
\hline$\# 18$ & 1.70 & 5.00 & 3.00 & $\begin{array}{l}\text { Intermediate-level } \\
\text { amplification }\end{array}$ & No & Normal \\
\hline$\# 19$ & 1.70 & 5.00 & 3.00 & $\begin{array}{l}\text { Intermediate-level } \\
\text { amplification }\end{array}$ & No & Normal \\
\hline \#20 & 1.25 & 5.00 & 4.00 & $\begin{array}{l}\text { Intermediate-level } \\
\text { amplification }\end{array}$ & Yes & Normal \\
\hline \#21 & 1.00 & 5.00 & 5.00 & $\begin{array}{l}\text { Intermediate-level } \\
\text { amplification }\end{array}$ & Yes & Normal \\
\hline \#22 & 1.25 & 5.00 & 4.00 & $\begin{array}{l}\text { Intermediate-level } \\
\text { amplification }\end{array}$ & Yes & Normal \\
\hline \#23 & 1.00 & 5.00 & 5.00 & $\begin{array}{l}\text { Intermediate-level } \\
\text { amplification }\end{array}$ & Yes & Normal \\
\hline \#24 & 1.25 & 5.00 & 4.00 & $\begin{array}{l}\text { Intermediate-level } \\
\text { amplification }\end{array}$ & Yes & Normal \\
\hline$\# 25$ & 1.00 & 5.00 & 5.00 & $\begin{array}{l}\text { Intermediate-level } \\
\text { amplification }\end{array}$ & Yes & Normal \\
\hline \#26 & 1.00 & 5.00 & 5.00 & $\begin{array}{l}\text { Intermediate-level } \\
\text { amplification }\end{array}$ & Yes & Normal \\
\hline \#27 & 1.00 & 5.00 & 5.00 & $\begin{array}{l}\text { Intermediate-level } \\
\text { amplification }\end{array}$ & Yes & Normal \\
\hline \#28 & 1.25 & 5.00 & 4.00 & $\begin{array}{l}\text { Intermediate-level } \\
\text { amplification }\end{array}$ & Yes & Normal \\
\hline \#29 & 0.83 & 5.00 & 6.00 & $\begin{array}{l}\text { Intermediate-level } \\
\text { amplification }\end{array}$ & Yes & Normal \\
\hline$\# 30$ & 1.26 & 5.00 & 4.00 & $\begin{array}{l}\text { Intermediate-level } \\
\text { amplification }\end{array}$ & Yes & Amplified \\
\hline \#31 & 1.50 & 4.90 & 3.20 & $\begin{array}{l}\text { Low-level } \\
\text { amplification }\end{array}$ & No & Amplified \\
\hline \#32 & 1.50 & 4.50 & 3.00 & $\begin{array}{c}\text { Low-level } \\
\text { amplification }\end{array}$ & No & Normal \\
\hline \#33 & 1.50 & 4.50 & 3.00 & $\begin{array}{l}\text { Low-level } \\
\text { amplification }\end{array}$ & No & Normal \\
\hline \#34 & 1.50 & 4.50 & 3.00 & $\begin{array}{l}\text { Low-level } \\
\text { amplification }\end{array}$ & No & Normal \\
\hline \#35 & 1.10 & 4.50 & 5.00 & $\begin{array}{l}\text { Low-level } \\
\text { amplification }\end{array}$ & Yes & Normal \\
\hline \#36 & 1.13 & 4.50 & 4.00 & $\begin{array}{l}\text { Low-level } \\
\text { amplification }\end{array}$ & Yes & Normal \\
\hline \#37 & 1.13 & 4.50 & 4.00 & $\begin{array}{c}\text { Low-level } \\
\text { amplification }\end{array}$ & Yes & Normal \\
\hline
\end{tabular}


Table 1. Cont.

\begin{tabular}{|c|c|c|c|c|c|c|}
\hline & & & FISH & & & NGS \\
\hline Case-ID & MET/CEN7 Ratio & MET GCN & CEN7 GCN & MET Status & Polysomy & NGS Status \\
\hline$\# 38$ & 1.33 & 4.00 & 3.00 & $\begin{array}{c}\text { Low-level } \\
\text { amplification }\end{array}$ & No & Normal \\
\hline \#39 & 1.30 & 4.00 & 3.00 & $\begin{array}{c}\text { Low-level } \\
\text { amplification }\end{array}$ & No & Normal \\
\hline$\# 40$ & 1.14 & 4.00 & 3.50 & $\begin{array}{c}\text { Low-level } \\
\text { amplification }\end{array}$ & No & Normal \\
\hline$\# 41$ & 1.00 & 4.00 & 4.00 & $\begin{array}{c}\text { Low-level } \\
\text { amplification }\end{array}$ & Yes & Normal \\
\hline$\# 42$ & 1.00 & 4.00 & 4.00 & $\begin{array}{c}\text { Low-level } \\
\text { amplification }\end{array}$ & Yes & Normal \\
\hline$\# 43$ & 1.00 & 3.50 & 3.50 & Normal & No & Amplified \\
\hline
\end{tabular}

a

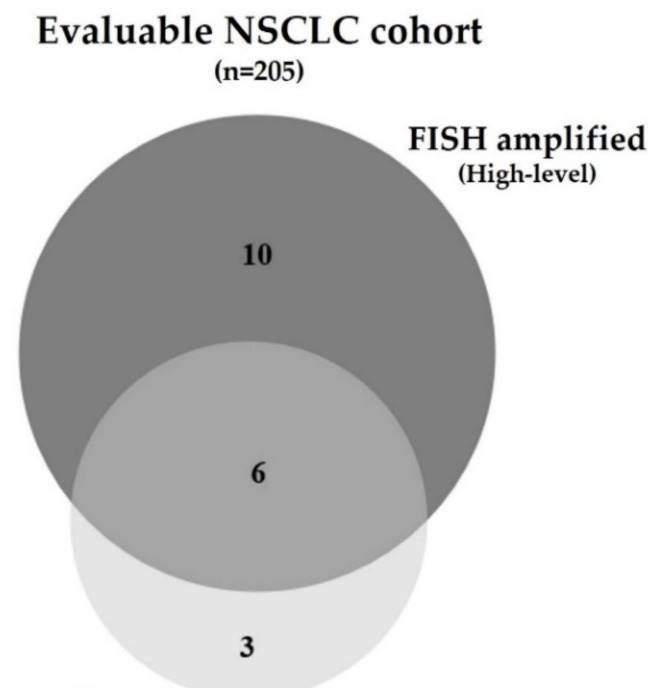

NGS amplified

$\mathrm{b}$

\section{Evaluable NSCLC cohort \\ $(n=205)$}

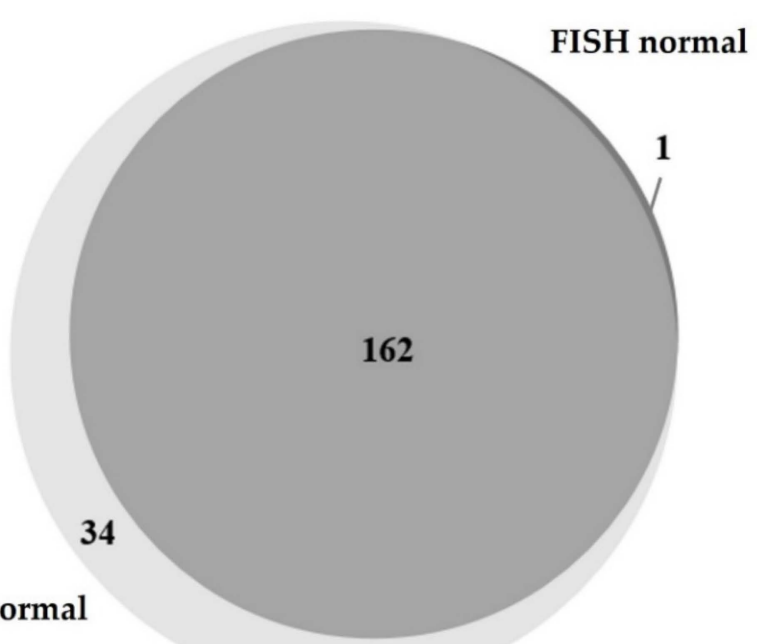

Figure 2. Comparison of the MET GCN of the NSCLC cohort using two different methods: FISH versus NGS. (a) Quantitative Venn Diagram of the evaluable NSCLC cases harboring a high-level amplification of the MET gene determined by either FISH (dark gray, polysomic cases included) or NGS (light gray, status according to the CRAFT algorithm). Overlaping cases are depicted in mid-gray color. (b) Quantitative Venn Diagram of the evaluable NSCLC cases showing no amplification of the MET gene determined by either FISH (dark gray) or NGS (light gray, status according to the CRAFT algorithm). Overlaping cases are depicted in mid-gray color.

Compared to these findings, $n=9$ cases were classified as MET gene amplified assessed by the NGS approach (Table 1 and Figure $2 \mathrm{a}$ ).

Comparing both methods regarding the status of the $M E T$ GCN revealed a discrepancy of $n=35 / 43$ cases $(81.4 \%)$. Focusing on both, the high-level MET amplified cases assessed by FISH and the MET amplified cases assessed by NGS showed a better concordance. Six of $n=16$ cases classified as high-level MET amplified by FISH were classified as MET amplified by NGS (37.5\%, cases \#2-6 and \#15, Figure 2a). Cases harboring a MET GCN of $>10.0$ showed the best concordance between FISH and NGS. 
This observation emphasizes that only cases harboring a high MET GCN or relatively high MET/CEN7 ratio classified by FISH can be detected adequately by the NGS approach. However, one of five cases was not classified as MET gene amplified by NGS.

Furthermore, looking at the overlap of $n=6$ cases, $n=2$ cases showed a concomitant polysomy determined by FISH. Case \#3 represents a patient with an EGFR mutation who had undergone EGFR TKI therapy for one year (see Material and Methods). The high-level amplification of the $M E T$ gene in this case likely represents a resistance mechanism as a consequence of EGFR TKI therapy.

The $n=10$ cases that were not classified as MET amplified by NGS but showed a clear high-level amplification by FISH demonstrates that not only polysomic MET amplified samples, but also cases harboring a high MET GCN >10 are not reliably detected by the NGS approach (Cases \#1, 7-14 and 16, Figure 2a). Case \#1, yielding a MET GCN >10 and a $M E T / C E N 7$ ratio $>5$, revealed a huge discrepancy between FISH and NGS. This case showed cluster formation of the MET signal assessed by FISH.

The $n=3$ cases that were classified as MET gene amplified by the NGS approach but were not defined as high-level amplified by FISH harbored an intermediate-/lowlevel amplification, or completely lacked amplification of MET (Cases \#30-31 and 43, Figure 2a). Case \#43 was classified as having the MET gene amplified by NGS but showed no MET amplification when assessed by FISH. The mean coverage of the MET locus in this particular case was 11.000, whereas the mean coverage of the CEN7 region was 27.300. With a normalized ratio of MET/CEN7 of 0.40 , calculated manually based on the amplicon coverages, the TST15-based algorithm defined this particular case as MET gene amplified for unknown reasons.

With regard to the normal, non-MET gene amplified cases, only $n=1$ case was classified as non-MET amplified by FISH but determined as MET amplified by NGS (Case \#43, Table 1 and Figure 2b). Furthermore, mainly intermediate- and low-level MET gene amplified cases were missed by the NGS approach (Figure 2b). Many of these cases showed a concomitant polysomy of the cells. For $n=162$ cases, both NGS and FISH approaches consistently classified the NSCLC cases as non-MET gene amplified (i.e., normal).

In summary, comparing FISH versus NGS regarding the status of the MET GCN revealed a discrepancy of $n=48$ of $n=205$ cases $(23.4 \%$, Figure 2a,b).

\subsection{Correlations of MET GCN with Patient Data}

Investigation of a potential correlation of an NGS-based identification of MET gene amplification and the sex and/or age of the patients in the cohort revealed a trend for an enriched amplification of MET in males (Figure 3a). No correlation was observed between MET gene amplification status and patient age (Figure 3b). Interestingly, the presence of MET gene amplification determined by NGS did not exclude a concurrent mutation in oncogenic driver genes. Focusing on the MET gene itself, our cohort demonstrates that MET gene amplification was never accompanied by a single-nucleotide variant (SNV) in the MET gene itself. 
a

\section{Sex}

-

MET $5 \%$ Аกับ KRAS $33 \%$ g. EGFR $20 \%$ ||

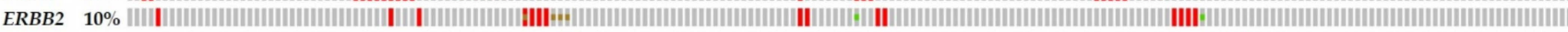

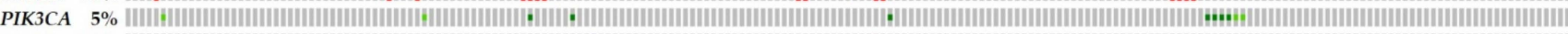

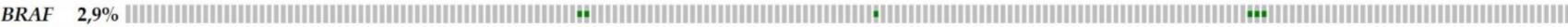
NRAS $1,5 \%$ V.

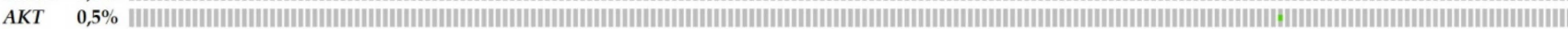

$$
\begin{aligned}
& \text { | } \text { Female } \mid \text { Male } \\
& \text { In-frame Mutation (VUS) } \| \text { Missense Mutation (Putative driver) } \| \text { Missense Mutation (VUS) } \mid \text { Amplification (VUS) } \mid \text { Wild-type }
\end{aligned}
$$

b

\section{Age}

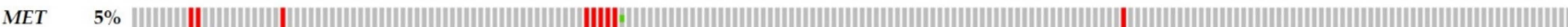

KRAS $33 \%$ || || || EGFR $20 \%$ | ERBB2 $10 \%$ i PIK3CA $5 \%$ | BRAF $2,9 \%$ |

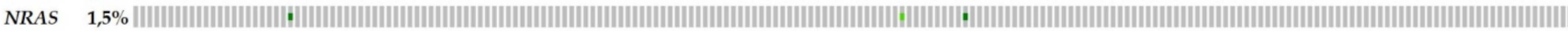
AKT $\quad 0,5 \%$ |

$$
\begin{aligned}
& \begin{array}{|l|l|l|l|l|l|l|l|}
0-30 & 31-40 & 41-50 & 51-60 & \text { | } 61-70 & 71-80 & 81-90 & 91-100
\end{array} \\
& \text { | In-frame Mutation (VUS) | Missense Mutation (Putative driver) || Missense Mutation (VUS) |Amplification (VUS) || Wild-type }
\end{aligned}
$$

Figure 3. Mutational profile of the consecutive NSCLC cohort regarding the sex and age of the patients. (a) Arrangement of the cases according to the sex of the patients. Each column represents one case. Pink color resembles females, whereas the blue color resembles males. (b) Arrangement of the cases according to the age of the patients. Each column represents one case. The various age groups are colored differently (dark blue $=0-30$, red $=31-40$, orange $=41-50$, dark green $=51-60$, violet $=61-70$, light blue $=71-80$, pink $=81-90$, light green $=91-100)$. In both $(\mathbf{a}, \mathbf{b})$ the affected genes are listed on the left with the frequency in the cohort shown as percent. Furthermore, in both $(\mathbf{a}, \mathbf{b})$, in-frame or missense mutations classified as variants of unknown significance (VUS) are labeled in brown and light green, respectively. Known missense mutations (putative driver mutations) are labeled in dark green. Cases harboring an alteration in gene copy numbers are depicted in red. Nonmutant, wild type samples are labeled in gray.

Amplifications of ERBB2 and EGFR, as well as somatic point mutations in the protooncogene KRAS, were observed (cases \#1, 3, 17, and 19). Case \#3 harbored an in-frame exon 19 deletion mutation in the EGFR gene. Case \#1 was characterized by a high-level MET amplification assessed by FISH, whereas case \#17 showed an intermediate-level amplification and case \#19 showed a normal level of the MET gene. Another notable point relates to the fact that somatic SNVs in the EGFR gene are often accompanied by amplification of the gene itself. In contrast, amplification of the ERBB2 or MET gene is usually not associated with a concomitant SNV at the DNA level.

\section{Discussion}

In this study, we directly compared $n=205$ consecutive NSCLC cases from routine diagnostics regarding the evaluation of MET GCN and the type of MET gene copy aberration using either an amplicon-based, 15-gene NGS panel or the standard FISH method. Out of the evaluable $n=205$ cases, $n=9$ cases $(4.4 \%)$ were classified as MET amplified by NGS. In contrast, $n=16$ cases were classified as high-level MET amplified by FISH (including polysomic cases). Only $n=6$ of the amplified cases, determined by NGS, were concurrently classified as MET amplified by FISH, yielding a discrepancy of $n=7(43.7 \%)$ cases. Focusing on the cases harboring a MET GCN $>10$ as assessed by FISH, the NGS approach reliably classified $80.0 \%$ of these cases as MET amplified $(n=4 / 5)$.

Our study shows for the first time that using the small focus gene panel TST15 from Illumina one is able to detect MET amplified cases, but not reliably. In line with previously published data, our study showed that using an NGS approach, high-level MET amplified 
cases (MET GCN >10.0, [13]) can be detected, even though not reliable. Various aspects of this study again emphasized that an exclusive NGS-based determination of the MET GCN cannot completely replace a FISH-based assessment of the MET gene status. Heydt et al. who used custom based NGS panels from Qiagen or an Ion AmpliSeq Custom panel from Thermo Fisher already faced similar problems [29]. They convincingly showed that MET IHC had the best concordance with MET FISH when comparing $n=35$ MET amplified samples (low-, intermediate- and high-level MET amplification). In contrast, the NanoString copy number assay, ddPCR copy number assay and custom amplicon-based parallel sequencing showed a lower concordance, especially when looking at the lowlevel MET-amplified samples. Furthermore, they showed that high-level MET-amplified cases generally showed better concordance between NGS and FISH than intermediateor low-level MET-amplified cases. They claim that only MET high-level amplified samples harboring a GCN $\geq 6$ determined by FISH can be detected via alternative methods, including NGS. Additionally, other groups such as Guo et al. and Clavé et al. already compared the specificity and sensitivity of various methods for the detection of MET gene amplification [30,31]. Guo et al. mainly focused on the question of whether a MET overexpression detected by IHC could be correlated to a MET gene amplification or MET exon 14 skipping. They conclude that IHC is not a useful method for the detection of genomic changes of the MET locus as with gene amplification or MET exon 14 skipping. We strictly focused on the amplification of the MET gene, comparing the two methods, FISH versus NGS. Moreover, two institutes performing NGS used a hybrid capture based assay, not a PCR amplicon-based one as we did in our analysis. Using a hybrid capture based NGS assay, one investigates a larger gene panel and could lose some information of certain gene loci. In contrast, amplicon sequencing has a higher on target rate yielding a high specificity and deep coverage.

In contrast to [31] their biased, non-consecutive approach selecting $n=26$ MET gene amplified (FISH; MET GCN >5, Cappuzzo Score) samples of in total $n=222$ NSCLC cases, we used an unbiased consecutive, double blinded approach. In addition, they used a 500+ NGS gene panel (PGDx elioTM) and set a cut off for MET gene amplification of $>3.0$-fold change. In our study, cases harboring a fold change $>1.6$ were classified as $M E T$ gene amplified by NGS. For assessment of MET amplification by FISH, they referred to two individual FISH score references (Cappuzzo score and UCCC FISH-criteria). In our study, we referred to only one FISH scoring system [13].

In our hands, the PCR amplicon based NGS approach was able to detect cases harboring a high MET GCN $>10$, even in the presence of low polysomy ( $n=2$ cases). However, the NGS approach was not able to detect the various facets of MET amplification. No certain $\log$ fold changes could be defined that would segregate intermediate- to low-level $M E T$ amplification. Only $n=2$ cases (Case \#30,31) harboring an intermediate- or low-level MET amplification assessed by FISH were also classified as amplified by NGS. In most cases, the NGS approach classified MET GCN as normal, although the corresponding FISH samples yielded intermediate- to low-level MET gene amplification.

Whether a discrimination between "true" high-level MET gene amplified cases and intermediate- to low-level MET amplified cases is of clinical relevance is still debated. "True" high-level MET-amplified cells are characterized by a high MET/CEN7 ratio without concomitant polysomy. In a retrospective study, it was investigated whether "true" high-level $M E T$ amplified cases responded more efficiently to targeted therapy than intermediate- to low-level MET amplified cases [32]. The study highlighted that only "true" high-level MET amplified cases harboring MET/CEN7 ratios $\geq 5.0$ efficiently responded to targeted therapy.

The discrepancy between the FISH and NGS approaches observed in the present study could be due to biological or technical reasons regarding the $M E T$ gene locus. For example, the mean coverage of the ERBB2 gene in the present study comprised 15.000 reads, whereas the average coverage of the $M E T$ gene comprised a far lower amount of less than 5.000 reads (data not shown). Although amplification of the MET gene takes place, the number of amplified reads could still be low and could impede PCR amplification and subsequent detection. 
Another issue concerns the tumor content and tumor purity of a sample. If the tumor content is too low, NGS might show false negative results, as the normal cell content could distort the results. Therefore, carefully concerted microdissection of the tumor area is crucial to avoid contamination with normal tissue [33].

From a technical point of view, the way the MET gene itself is organized on the chromosome could be problematic. The ladder-like structure characterized by many inverted repeats makes the gene locus prone to gene amplification but may also complicate sophisticated primer design [5]. Perhaps the primer-annealing efficiency in these regions is impaired, leading to lower coverage than expected. In addition, a high content of the two nucleotides guanine and cytosine (GC content) of the MET locus could impair appropriate dissociation of the DNA strands during the PCR denaturation step.

Our study also highlights the limitations of the NGS approach with regard to the polysomic state of the cells. For example, cases \#7, 8 and 10-12 showed a high-level amplification of the MET gene assessed by FISH, whereas the NGS method classified these cases as normal. These $n=5$ cases were all polysomic, thus not resembling the "true" highlevel MET amplified samples. Looking at the coverage of the corresponding NGS amplicon files revealed high coverage values of the CEN7 control region and lower coverage values for the corresponding MET locus. This observation could suggest that the TST15 CRAFT algorithm missed these high-level MET amplified cases, probably due to a defined cut-off, which depends on the calculation of the median normalized bin count of the target gene versus the median bin count of the entire panel. In the case of a polysomic state of the cell, $M E T$ amplification could be masked.

Using the corresponding normal healthy tissue of a tumor sample as a reference for the calculation of the ratios and a normal reference pool could circumvent such a problem. Grasso et al. used a PCR amplicon-based approach and developed an algorithm for the assessment of GCN alterations that used a sequence data pool of normal samples as a reference [33]. They convincingly showed that they detected clinically relevant GCN alterations (i.e., ERBB2, EGFR, MET) for $n=14$ breast cancer samples using the matched normal samples or a normal reference pool. Similarly, Niu et al. defined an ERBB2 breast cancer-specific cutoff for the NGS algorithm by sequencing $n=151$ ERBB2 nonamplified FFPE samples [34]. By this approach, they also created a normal reference pool. With the aid of using a normal reference pool, the sensitivity of the NGS GCN determination could be increased, as a polysomic state of the cells could not mask the detection of a possible GCN alteration. Moreover, one could save money by avoiding sequencing the matching normal tissue of a tumor specimen.

Another critical disadvantage for the assessment of MET amplification by NGS is that cells harboring cluster formation of the MET gene locus can be sparse and therefore can be masked by surrounding tumor cells without cluster formation (see case \#1, Table 1). The NGS-based algorithm might miss such cells and, hence, would define such samples as false negatives.

Another limitation of the TST15 NGS approach is that the customer does not receive any detailed information on ratios or total gene copy numbers. Although the newer gene panels TST170 or TSO500 (both Illumina) make use of the same CRAFT gene copy number algorithm, these new methods offer the customer values of fold changes and a possibility to determine the total gene copy number of a gene of interest. Furthermore, other technologies, such as the FoundationOne Assay, used in the study of Frampton et al., provide the customer with total gene copy number values. In this particular case, they defined samples harboring $\geq 6.0$ gene copies of the $M E T$ gene as amplified [23].

Our study also focused on a possible correlation between the sex and age of the patients and concomitant MET gene amplification determined by NGS. The trend of more males having an amplification of the MET gene is in line with data from Okuda et al., showing that in a cohort of $n=213$ NSCLC cases ( $n=148$ males and $n=65$ females), all MET amplified cases were males [35]. Although these males were all smokers, hindering a clear correlation between sex and MET gene amplification, smoking behavior could also 
lead to the observed phenotype. Information concerning female smokers is lacking in this particular case.

Another interesting issue, which was addressed by the present study, was the occurrence of concomitant driver gene mutations in the presence of $M E T$ amplification. In our study, $n=4$ out of the $n=9$ MET gene amplified cases determined by NGS showed a concomitant molecular alteration in the genes KRAS, EGFR and ERBB2. Focusing on the "true" high-level MET amplified cases classified by FISH, $n=6$ of $n=9$ cases showed a concurrent mutation of other oncogenic driver genes. These results are against the common consensus in the literature that a "true" high-level MET amplification is not simultaneously accompanied by a mutation in one of the known driver genes. The study of Noonan et al. revealed that the "true" high-level MET amplified cases explicitly did not show a concomitant mutation in another oncogenic driver gene, such as KRAS or EGFR [32]. Looking in more detail, case \#15 of our study was classified as high-level MET amplified by the FISH approach but revealed only a MET GCN of 4.4 accompanied by a GCN of CEN7 of 1.6 leading to a ratio of 2.7. Based on the classification system according to [13], this case is classified as high-level MET amplified but probably does not reflect a "true" high-level MET amplified case.

Regarding the MET gene itself, our cohort did not include cases that showed a concurrent mutation of the MET gene in the presence of $M E T$ gene amplification. An investigation of a cohort of $n=178$ NSCLC cases by NGS showed that the MET gene itself is rarely mutated, and only $n=3$ cases showed a somatic exon 14 deleting splice-site mutation [35]. Currently, there are no data that directly address the question of whether the amplification of the MET gene impedes a concurrent mutation of the gene itself.

Our rate of clinically relevant "true" high-level MET amplification (4.4\%) using FISH is similar to the observed rate in the study by Okuda et al., where they obtained a rate of $5,6 \%$ MET gene amplified cases of $n=213$ NSCLC patients [35]. Tsuta et al. described more divergent findings compared to our study cohort reporting amplification of the MET gene in 10,9\% of NSCLC cases assessed by BISH (bright-field in situ hybridization, BISH-positive, when one of five criteria were met, for example: MET to CEN7 ratio $\geq 2.0$ or $>15.0$ copies of the MET signal in $>10 \%$ of tumor cells) [36]. On the other hand, when comparing our study with the results obtained by Park et al., our rate of MET gene amplification was higher compared to their results showing MET amplification in $2.4 \%$ of $n=380$ NSCLC cases determined by FISH using the University of Colorado Cancer Center criteria (UCCCC) [37]. Using the Capuzzo scoring system, the authors obtained a rate of $7.1 \%$ MET gene amplified cases. Using MET IHC, they found that $13.7 \%$ of patients showed MET overexpression. In another study that investigated a large cohort of patients with surgically resected NSCLC for MET overexpression and gene amplification, Sterlacci et al. found MET amplification in $2.4 \%$ of cases assessed by FISH using tissue microarrays (using a defined cut-off for amplifications of $M E T$ to $C E N 7$ signal ratio of $\geq 2.0$ ) [38]. Schildhaus et al. described $3 \%$ of NSCLC being clear-cut MET high-level amplified. However, low- and intermediate-level amplifications were assessed in 30\% of investigated NSCLCs [13]. In summary, these data show that the comparability between different laboratories is difficult and that the interpretation of $M E T$ amplification regarding the prognostic value and the various levels of $M E T$ amplification, respectively, is challenging.

The disadvantages of our study comprise the partly retrospective character of the study (assessment of MET FISH). Moreover, we were not able to directly link the MET GCN alteration status with smoking behavior and prognosis of the respective NSCLC patients. In addition, we were not able to monitor whether these patients obtained targeted therapy. One reason for this lack of information is that some cases had been sent from external pathologies. Another point of criticism is the fact that $32.7 \%$ of our cohort could not be evaluated using MET FISH due to insufficient quality of the fluorescence signal, limited amount of material, or lack of the corresponding material. These limitations were also encountered in other studies where for example silver in-situ hybridization (SISH) was not assessable in $15 \%$ of patients [39]. Regarding NGS, data were evaluable in $93 \%$ 
of our cohort. Only $7 \%$ of the samples could not be analyzed via NGS due to insufficient amount of the material or low coverage of the MET gene locus. This highlights a positive aspect of the NGS method as-although material can be sparse-the PCR amplification step and the highly sensitive sequencing platform make it possible to analyze a specimen, which could be potentially problematic for FISH.

In summary, our data agrees with previous studies in that a "true" high-level MET amplification is still a rare event in NSCLC tumorigenesis. In the near future, however, it could become more prominent as a potential secondary resistance mutation in response to an increasing number of prescribed tyrosine kinase inhibitor therapies. Therefore, the need for a harmonized scoring system using FISH, which enables a comparison between laboratories and the dissection of high-, intermediate- and low-level amplified cases, is still crucial. In addition, one should be aware of the limitations that arise when MET GCNs or GNCs in general are assessed by NGS. The use of normal reference pools in the case of MET GCN determination should be carefully considered, as nonclinical relevant high-level polysomic cases could also be included in this way of therapy stratification. Of interest, TKI-induced, resistance-associated amplification of the MET gene is not exclusively characterized by high-level MET amplification, as intermediate- to low-level amplifications are also observed [16]. Therefore, FISH-based determination of MET GCN is currently inevitably necessary to detect the various facets of $M E T$ gene amplifications.

\section{Conclusions}

The findings of the present study are of great importance, as the future of routine molecular diagnostics will be mainly based on NGS data. As gene amplifications can be obtained easily by NGS, one would be tempted to avoid a time- and tissue-consuming additional method such as FISH. However, NGS does not resemble a reliable substitute for the assessment of $M E T$ gene copy number. Using FISH during routine diagnostics for the assessment of the $M E T$ gene copy number will remain necessary to reliably detect the various levels of $M E T$ gene amplification.

Author Contributions: C.S., R.E., F.H. and A.H. designed and conceived the study. F.F., H.S., R.S.-B., R.L., M.K. and W.H.-S. assigned the patients and acquired and interpreted the data. G.S., W.S., M.V., C.S. (Christoph Seidl) and M.M. stated the diagnosis and acquired data. R.E. analyzed the FISH experiments. C.S. (Christoph Schubart), L.T. and R.S. performed and analyzed the NGS experiments. C.S. (Christoph Schubart) and R.E. analyzed data and generated figures. C.S. (Christoph Schubart) and R.E. wrote the original draft of the manuscript. All authors have read and agreed to the published version of the manuscript.

Funding: This research was partially funded by external research funding (Novartis Oncology). Furthermore, this work was supported by the Interdisciplinary Center for Clinical Research (IZKF, Clinician Scientist Program) of the Medical Faculty FAU Erlangen-Nürnberg.

Institutional Review Board Statement: Ethical review and approval were obtained by the local ethics committee, Friedrich-Alexander-Universität Erlangen-Nürnberg, Krankenhausstraße 12, 91054 Erlangen (Proposal number 85_17 B).

Informed Consent Statement: Patient consent was waived due to administration issues regarding the retrospective analysis of some FISH cases.

Data Availability Statement: Data can be obtained on request. Data are not stored on publicly available servers.

Acknowledgments: We thank Susanne Blank for excellent technical support regarding the FISH experiments. We thank Simone Hebele for excellent technical support regarding the NGS experiments.

Conflicts of Interest: A.H. has received honoraria from BMS, MSD, Roche, AstraZeneca, Boehringer Ingelheim, Abbvie, Jansen-Cilag, and Ipsen. R.E. has received honoraria from Roche, Eisai, Pfizer, and Novartis and travel grants from BioNTech. The institution of A.H. and R.E. conducts research for AstraZeneca, Roche, Janssen-Cilag, NanoString Technologies, Biocartis, Zytomed Systems, Novartis, Cepheid, and BioNTech. R.S.B. received honoraria from BMS, Roche, MSD, Novartis, Celgene, 
Amgen and Ipsen. W.S. and M.V. conduct research/received honoraria for/from Falk, Pentax, Lilly, Malesci, AstraZeneca, Olympus, Leica, Rohe, Boerhinger-Ingelheim.

\section{References}

1. Cohen, N.; Kim, T.; DeMatteo, R.P. Principles of Kinase Inhibitor Therapy for Solid Tumors. Ann. Surg. 2017, 265, 311-319. [CrossRef]

2. Organ, S.L.; Tsao, M.-S. An overview of the c-MET signaling pathway. Ther. Adv. Med Oncol. 2011, 3 (Suppl. 1), S7-S19. [CrossRef] [PubMed]

3. Ma, P.C.; Maulik, G.; Christensen, J.; Salgia, R. c-Met: Structure, functions and potential for therapeutic inhi-bition. J. Cancer Metastasis Rev. 2003, 22, 309-325. [CrossRef]

4. Tong, J.H.; Yeung, S.F.; Chan, A.; Chung, L.Y.; Chau, S.L.; Lung, R.W.M.; Tong, C.Y.; Chow, C.; Tin, E.K.; Yu, Y.H.; et al. MET Amplification and Exon 14 Splice Site Mutation Define Unique Molecular Subgroups of Non-Small Cell Lung Carcinoma with Poor Prognosis. Clin. Cancer Res. 2016, 22, 3048-3056. [CrossRef] [PubMed]

5. Hellman, A.; Zlotorynski, E.; Scherer, S.W.; Cheung, J.; Vincent, J.B.; Smith, D.I.; Trakhtenbrot, L.; Kerem, B. A role for common fragile site induction in amplification ofhuman oncogenes. Cancer Cell 2002, 1, 89-97. [CrossRef]

6. AACR Project Genie Consortium. AACR Project GENIE: Powering Precision Medicine through an International Consor-tium. Cancer Discov. 2017, 7, 818-831. [CrossRef]

7. Cappuzzo, F.; Marchetti, A.; Skokan, M.; Rossi, E.; Gajapathy, S.; Felicioni, L.; Del Grammastro, M.; Sciar-rotta, M.G.; Buttitta, F.; Incarbone, M.; et al. Increased MET gene copy number negatively affects survival of surgically resected non-small-cell lung cancer patients. J. Clin. Oncol. Off. J. Am. Soc. Clin. Oncol. 2009, 27, 1667-1674. [CrossRef]

8. Go, H.; Jeon, Y.K.; Park, H.J.; Sung, S.-W.; Seo, J.-W.; Chung, D.H. High MET Gene Copy Number Leads to Shorter Survival in Patients with Non-small Cell Lung Cancer. J. Thorac. Oncol. 2010, 5, 305-313. [CrossRef]

9. Chen, H.J.; Mok, T.S.; Chen, Z.H.; Guo, A.L.; Zhang, X.C.; Su, J.; Wu, Y.L. Clinicopathologic and molecu-lar features of epidermal growth factor receptor T790M mutation and c-MET amplification in tyrosine kinase inhibitor-resistant Chinese non-small cell lung cancer. Pathol. Oncol. Res. 2009, 15, 651-658. [CrossRef]

10. Bean, J.; Brennan, C.; Shih, J.-Y.; Riely, G.; Viale, A.; Wang, L.; Chitale, D.; Motoi, N.; Szoke, J.; Broderick, S.; et al. MET amplification occurs with or without T790Mmutations in EGFR mutant lung tumors with acquired resistance to gefitinib or erlotinib. Proc. Natl. Acad. Sci. USA 2007, 104, 20932-20937. [CrossRef]

11. Campbell, J.D.; Alexandrov, A.; Kim, J.; Wala, J.; Berger, A.H.; Pedamallu, C.S.; Shukla, S.A.; Guo, G.; Brooks, A.N.; Murray, B.A.; et al. Distinct patterns of somatic genome alterations in lung adenocarci-nomas and squamous cell carcinomas. Nat. Genet. 2016, 48, 607. [CrossRef]

12. Rieger, R.; Michaelis, A.; Green, M.M. A Glossary of Genetics and Cytogenetics: Classical and Molecular; Springer: New York, NY, USA, 1968.

13. Schildhaus, H.-U.; Schultheis, A.M.; Rüschoff, J.; Binot, E.; Merkelbach-Bruse, S.; Fassunke, J.; Schulte, W.; Ko, Y.-D.; Schlesinger, A.; Bos, M.; et al. MET Amplification Status in Therapy-Naïve Adeno- and Squamous Cell Carcinomas of the Lung. Clin. Cancer Res. 2014, 21, 907-915. [CrossRef] [PubMed]

14. Wu, Y.L.; Zhang, L.; Kim, D.W.; Liu, X.; Lee, D.H.; Yang, J.C.; Ahn, M.J.; Vansteenkiste, J.F.; Su, W.C.; Felip, E.; et al. Phase Ib/II Study of Capmatinib (INC280) Plus Gefitinib After Failure of Epidermal Growth Factor Receptor (EGFR) Inhibitor Ther-apy in Patients With EGFR-Mutated, MET Factor-Dysregulated Non-Small-Cell Lung Cancer. J. Clin. Oncol. 2018, 36, 3101-3109. [CrossRef]

15. Planchard, D.; Loriot, Y.; André, F.; Gobert, A.; Auger, N.; Lacroix, L.; Soria, J.C. EGFR-independent mecha-nisms of acquired resistance to AZD9291 in EGFR T790M-positive NSCLC patients. Ann. Oncol. 2015, 26, 2073-2078. [CrossRef]

16. Dagogo-Jack, I.; Yoda, S.; Lennerz, J.K.; Langenbucher, A.; Lin, J.J.; Rooney, M.M.; Prutisto-Chang, K.; Oh, A.; Adams, N.A.; Yeap, B.Y.; et al. MET Alterations Are a Recurring and Ac-tionable Resistance Mechanism in ALK-Positive Lung Cancer. Clin. Cancer Res. 2020, 26, 2535-2545. [CrossRef] [PubMed]

17. Ou, S.-H.I.; Kwak, E.L.; Siwak-Tapp, C.; Dy, J.; Bergethon, K.; Clark, J.W.; Camidge, D.R.; Solomon, B.J.; Maki, R.G.; Bang, Y.-J.; et al. Activity of Crizotinib (PF02341066), a Dual Mesenchymal-Epithelial Transition (MET) and Anaplastic Lymphoma Ki-nase (ALK) Inhibitor, in a Non-small Cell Lung Cancer Patient with De Novo MET Amplification. J. Thor. Oncol. 2011, 6, 942-946. [CrossRef] [PubMed]

18. Camidge, D.R.; Ou, S.-H.I.; Shapiro, G.; Otterson, G.A.; Villaruz, L.C.; Villalona-Calero, M.A.; Iafrate, A.J.; Varella-Garcia, M.; Dacic, S.; Cardarella, S.; et al. Efficacy and safety of crizotinib in patients with advanced c-MET-amplified non-small cell lung cancer (NSCLC). J. Clin. Oncol. 2014, 32, 8001. [CrossRef]

19. Wolf, J.; Seto, T.; Han, J.Y.; Reguart, N.; Garon, E.B.; Groen, H.; Tan, D.S.W.; Hida, T.; de Jonge, M.; Orlov, S.V.; et al. Capmatinib in MET Exon 14-Mutated or MET-Amplified Non-Small-Cell Lung Cancer. N. Engl. J. Med. 2020, 383, 944-957. [CrossRef]

20. Turke, A.B.; Zejnullahu, K.; Wu, Y.-L.; Song, Y.; Dias-Santagata, D.; Lifshits, E.; Toschi, L.; Rogers, A.; Mok, T.; Sequist, L.; et al. Preexistence and Clonal Selection of MET Amplifi-cation in EGFR Mutant NSCLC. Cancer Cell 2010, 17, 77-88. [CrossRef]

21. Mehta, A.; Vasudevan, S.; Sharma, S.K.; Panigrahi, M.; Suryavanshi, M.; Saifi, M.; Batra, U. Biomarker testing for advanced lung cancer by next-generation sequencing; a valid method to achieve a comprehensive glimpse at mutational landscape. Appl. Cancer Res. 2020, 40,1-12. [CrossRef] 
22. Jiang, R.; Zhang, B.; Teng, X.; Hu, P.; Xu, S.; Zheng, Z.; Liu, R.; Tang, T.; Ye, F. Validating a targeted next-generation sequencing assay and profiling somat-ic variants in Chinese non-small cell lung cancer patients. Sci. Rep. 2020, 10, 2070. [CrossRef]

23. Frampton, G.M.; Fichtenholtz, A.; Otto, G.A.; Wang, K.; Downing, S.R.; He, J.; Schnall-Levin, M.; White, J.; Sanford, E.M.; An, P.; et al. Development and validation of a clinical cancer genomic profil-ing test based on massively parallel DNA sequencing. Nat. Biotechnol. 2013, 31, 1023-1031. [CrossRef]

24. Viailly, P.-J.; Sater, V.; Viennot, M.; Bohers, E.; Vergne, N.; Berard, C.; Dauchel, H.; Lecroq, T.; Celebi, A.; Ruminy, P.; et al. Improving high-resolution copy number variation analysis from next generation sequencing using unique molecular identifiers. BMC Bioinform. 2021, 22, 120. [CrossRef] [PubMed]

25. Buckingham, L.E.; Coon, J.S.; Morrison, L.E.; Jacobson, K.K.; Jewell, S.S.; Kaiser, K.A.; Mauer, A.M.; Muzzafar, T.; Polowy, C.; Basu, S.; et al. The Prognostic Value of Chromosome 7 Polysomy in Non-small Cell Lung Cancer Patients Treated with Gefitinib. J. Thorac. Oncol. 2007, 2, 414-422. [CrossRef] [PubMed]

26. Hulsen, T.; Vlieg, J.d.; Alkema, W. BioVenn-A web application for the comparison and visualization of bio-logical lists using area-proportional Venn diagrams. BMC Genom. 2008, 9, 488. [CrossRef] [PubMed]

27. Cerami, E.; Gao, J.; Dogrusoz, U.; Gross, B.E.; Sumer, S.O.; Aksoy, B.A.; Jacobsen, A.; Byrne, C.J.; Heuer, M.L.; Larsson, E.; et al. The cBio Cancer Genomics Portal: An Open Platform for Exploring Multidimensional Cancer Genomics Data: Figure 1. Cancer Discov. 2012, 2, 401-404. [CrossRef] [PubMed]

28. Gao, J.; Aksoy, B.A.; Dogrusoz, U.; Dresdner, G.; Gross, B.; Sumer, S.O.; Sun, Y.; Jacobsen, A.; Sinha, R.; Larsson, E.; et al. Integrative Analysis of Complex Cancer Genomics and Clinical Profiles Using the cBioPortal. Sci. Signal. 2013, 6, pl1. [CrossRef]

29. Heydt, C.; Becher, A.-K.; Wagener-Ryczek, S.; Ball, M.; Schultheis, A.M.; Schallenberg, S.; Rüsseler, V.; Büttner, R.; MerkelbachBruse, S. Comparison of in situ and extraction-based methods for the detection of MET amplifications in solid tumors. Comput. Struct. Biotechnol. J. 2019, 17, 1339-1347. [CrossRef]

30. Guo, R.; Berry, L.D.; Aisner, D.L.; Sheren, J.; Boyle, T.; Bunn, P.A., Jr.; Johnson, B.E.; Kwiatkowski, D.J.; Drilon, A.; Sholl, L.M.; et al. MET IHC is a Poor Screen for MET Amplification or MET exon 14 muta-tions in Lung Adenocarcinomas: Data from a Tri-Institutional Cohort of the Lung Cancer Mutation Consortium. J. Thorac. Oncol. 2019, 14, 1666-1671. [CrossRef]

31. Clavé, S.; Salido, M.; Rocha, P.; Hardy-Werbin, M.; Gibert, J.; Riera, X.; Weingartner, E.; Cerqueira, C.; Nichol, D.; Simmons, J.; et al. 1991P-Identification of MET gene amplifications using next-generation sequencing in non-small cell lung cancer patients. Ann. Oncol. 2019, 30, v800. [CrossRef]

32. Noonan, S.A.; Berry, L.; Lu, X.; Gao, D.; Barón, A.E.; Chesnut, P.; Sheren, J.; Aisner, D.L.; Merrick, D.; Doebele, R.C.; et al. Identifying the Appropriate FISH Criteria for Defining MET Copy Number-Driven Lung Adenocarcinoma through Oncogene Overlap Analysis. J. Thorac. Oncol. 2016, 11, 1293-1304. [CrossRef] [PubMed]

33. Grasso, C.; Butler, T.; Rhodes, K.; Quist, M.; Neff, T.L.; Moore, S.; Tomlins, S.A.; Reinig, E.; Beadling, C.; Andersen, M.; et al. Assessing Copy Number Alterations in Targeted, Amplicon-Based Next-Generation Sequencing Data. J. Mol. Diagn. 2014, 17, 53-63. [CrossRef]

34. Niu, D.; Li, L.; Yu, Y.; Zang, W.; Li, Z.; Zhou, L.; Jia, L.; Rao, G.; Gao, L.; Cheng, G.; et al. Evaluation of Next Generation Sequencing for Detecting HER2 Copy Number in Breast and Gastric Cancers. Pathol. Oncol. Res. 2020, 26, 2577-2585. [CrossRef]

35. Okuda, K.; Sasaki, H.; Yukiue, H.; Yano, M.; Fujii, Y. Met gene copy number predicts the prognosis for com-pletely resected non-small cell lung cancer. Cancer Sci. 2008, 99, 2280-2285. [CrossRef] [PubMed]

36. Tsuta, K.; Kozu, Y.; Mimae, T.; Yoshida, A.; Kohno, T.; Sekine, I.; Tamura, T.; Asamura, H.; Furuta, K.; Tsuda, H. c-MET/PhosphoMET Protein Expression and MET Gene Copy Number in Non-small Cell Lung Carcinomas. J. Thor. Oncol. 2012, 7, 331-339. [CrossRef]

37. Park, S.; Choi, Y.-L.; Sung, C.O.; An, J.; Seo, J.; Ahn, M.-J.; Ahn, J.S.; Park, K.; Shin, Y.K.; Erkin, O.C.; et al. High MET copy number and MET overexpression: Poor outcome in non-small cell lung cancer patients. Histol. Histopathol. 2012, 27, 197-207. [CrossRef] [PubMed]

38. Sterlacci, W.; Fiegl, M.; Gugger, M.; Bubendorf, L.; Savic, S.; Tzankov, A. MET overexpression and gene amplification: Prevalence, clinico-pathological characteristics and prognostic significance in a large cohort of patients with surgically resected NSCLC. Virchows Arch. 2017, 471, 49-55. [CrossRef]

39. Dziadziuszko, R.; Wynes, M.W.; Singh, S.; Asuncion, B.R.; Ranger-Moore, J.; Konopa, K.; Rzyman, W.; Szostakiewicz, B.; Jassem, J.; Hirsch, F.R. Correlation between MET Gene Copy Number by Silver In Situ Hy-bridization and Protein Expression by Immunohistochemistry in Non-small Cell Lung Cancer. J. Thor. Oncol. 2012, 7, 340-347. [CrossRef] 